\title{
The effects of chronic trans-resveratrol supplementation on aspects of cognitive function, mood, sleep, health and cerebral blood flow in healthy, young humans
}

\author{
Emma L. Wightman ${ }^{1}$, Crystal F. Haskell-Ramsay ${ }^{1}$, Jonathon L. Reay ${ }^{2}$, Gary Williamson ${ }^{3}$, Tristan Dew ${ }^{3,4}$, \\ Wei Zhang ${ }^{3}$ and David O. Kennedy ${ }^{1}$ \\ ${ }^{1}$ Brain, Performance and Nutrition Research Centre, Northumbria University, Newcastle upon Tyne NE1 8ST, UK \\ ${ }^{2}$ School of Social Sciences and Law, Teesside University, Middlesbrough TS1 3BA, UK \\ ${ }^{3}$ School of Food Science and Nutrition, University of Leeds, Leeds LS2 9JT, UK \\ ${ }^{4}$ Bradford School of Pharmacy, School of Life Sciences, University of Bradford, Bradford BD7 1DP, UK \\ (Submitted 18 February 2015 - Final revision received 16 July 2015 - Accepted 17 July 2015 - First published online 7 September 2015)
}

\section{Abstract}

Single doses of resveratrol have previously been shown to increase cerebral blood flow (CBF) with no clear effect on cognitive function or mood in healthy adults. Chronic resveratrol consumption may increase the poor bioavailability of resveratrol or otherwise potentiate its psychological effects. In this randomised, double-blind, placebo-controlled, parallel-groups study, a total of sixty adults aged between 18 and 30 years received either placebo or resveratrol for $28 \mathrm{~d}$. On the 1 st and 28th day of treatment, the performance of cognitively demanding tasks (serial subtractions, rapid visual information processing and 3-Back) ( $n 41$ complete data sets) was assessed, alongside blood pressure ( $n$ 26) and acute (near-IR spectroscopy (NIRS)) and chronic (transcranial Doppler) measures of CBF ( $n$ 46). Subjective mood, sleep quality and health questionnaires were completed at weekly intervals ( $n$ 53/54). The results showed that the cognitive effects of resveratrol on day 1 were restricted to more accurate but slower serial subtraction task performance. The only cognitive finding on day 28 was a beneficial effect of resveratrol on the accuracy of the 3-Back task before treatment consumption. Subjective ratings of 'fatigue' were significantly lower across the entire $28 \mathrm{~d}$ in the resveratrol condition. Resveratrol also resulted in modulation of CBF parameters on day 1 , as assessed by NIRS, and significantly increased diastolic blood pressure on day 28. Levels of resveratrol metabolites were significantly higher both before and after the day's treatment on day 28 , in comparison with day 1 . These results confirm the acute CBF effects of resveratrol and the lack of interpretable cognitive effects.

\section{Key words: Resveratrol: Near-IR spectroscopy: Cerebral blood flow: Nitric oxide: Cognition}

Resveratrol $\left(3,4^{\prime}, 5\right.$ trihydroxystilbene) is a polyphenolic secondary metabolite produced within plants in response to a range of environmental stressors ${ }^{(1)}$. Previous investigations in young, healthy humans have demonstrated significantly increased cerebral blood flow (CBF) after acute resveratrol supplementation $^{(2)}$, which is probably mediated by the ability of resveratrol to modulate nitric oxide synthesis ${ }^{(3)}$. In line with this, oral consumption has been shown to enhance endothelium-dependent relaxation in rats ${ }^{(4,5)}$, and to improve flow-mediated dilatation in overweight/obese humans ${ }^{(6)}$. An increase in blood-borne neural metabolic substrates such as oxygen $^{(7)}$ and glucose ${ }^{(8)}$ has been shown to enhance aspects of cognitive performance in healthy, young humans. However, to date, there is no evidence that cognitive function is modulated during acute, resveratrol-mediated increases in $\mathrm{CBF}$.
One potential explanation for this lack of cognitive effects is the rapid metabolism and poor bioavailability of oral resveratrol ${ }^{(9)}$, which might reduce its potential bioactivity. Pharmacokinetic studies have demonstrated plasma $C_{\max }$ levels of resveratrol metabolites between 0.9 and $3.7 \mu$ following a single oral dose of $500 \mathrm{mg}$ of resveratrol ${ }^{(10)}$, with levels of the parent compound at trace or undetectable concentrations ${ }^{(2,10-13)}$ after acute, bolus supplementation. Conversely, results from three preclinical chemopreventive efficacy papers suggest that repeated low daily doses of resveratrol (up to $2 \mathrm{mg} / \mathrm{kg}$ ) are sufficient to produce peak plasma concentrations of aglycone resveratrol of up to $2 \mu \mathrm{M}$, potentially exerting beneficial chemopreventive effects ${ }^{(14)}$ possibly as a result of a cumulative increase in plasma levels of resveratrol.

Thus, the current study investigated the effects of 28-d supplementation with $500 \mathrm{mg}$ of resveratrol in healthy adults, with

Abbreviations: BP, blood pressure; CBF, cerebral blood flow; CBFV, CBF velocity; CW-NIRS, continuous-wave near-IR spectroscopy; deoxy-Hb, deoxygenated Hb; GHQ, General Health Questionnaire; MCA, middle cerebral artery; NIRS, near-IR spectroscopy; oxy-Hb, oxygenated HB; POMS, Profile of Mood States; PSQI, Pittsburgh Sleep Quality Inventory; TCD, transcranial Doppler.

* Corresponding author: E. L. Wightman, email emma.1.wightman@northumbria.ac.uk 
the hypothesis being that daily consumption of this polyphenol, over an extended period, may increase bioavailability in terms of plasma levels and may potentiate any effects on cognitive performance and $\mathrm{CBF}$. In the current study, continuous-wave near-IR spectroscopy (CW-NIRS) was used to monitor acute changes in $\mathrm{CBF}$ in the prefrontal cortex during the performance of cognitive tasks that activate this brain region. This technique was combined with transcranial Doppler (TCD) sonography, applied to the middle cerebral artery (MCA), which provides a measure of acute and chronic changes in global CBF velocity (CBFV) and which has been converged successfully with NIRS previously $^{(15)}$. Resveratrol has previously been shown to interact with a number of diffuse, health-related parameters such as antioxidant and anti-inflammatory status ${ }^{(16,17)}$, monoamine oxidase-A and B (MAO-A/B) $\operatorname{activity}^{(18)}$ and peroxisome proliferator-activated receptor gamma coactivator 1-alpha PGC- $1 \alpha^{(19)}$ production. Hence, the current study also assessed health, mood and sleep parameters via questionnaires.

\section{Methods}

\section{Participants}

All participants reported themselves to be in good health and free from illicit drugs, alcohol, prescription medication and herbal extracts/food supplements at each assessment. Participants confirmed that they would also abstain from the latter for the duration of the study and that any changes in medication or health status would be reported to the researcher when they occurred. Participants who had suffered a head injury, neurological disorder or neuro-developmental disorder were excluded from participation, as were those who did not have English as their first language, or had any relevant food allergies or intolerances, digestive problems, smoked tobacco, drank excessive amounts of caffeine (more than $600 \mathrm{mg} / \mathrm{d}$ as assessed by a caffeine consumption questionnaire), took illicit social drugs, were pregnant, seeking to become so or were breast-feeding.

The study received ethical approval from the Northumbria University Psychology Department (within the Faculty of Health and Life Sciences) ethics committee (reference: SUB16_EW_1010; date approved 11 November 2010) and was conducted according to the Declaration of Helsinki (1964). All participants gave their written informed consent before their inclusion in the study.

See Table 1 for participant composition (broken down per analysis).

\section{Treatments}

Over the course of this 28-d supplementation study, participants received either $500 \mathrm{mg}$ of pure trans-resveratrol (Transmax $^{\mathrm{TM}}$ by Biotivia ${ }^{\mathrm{TM}}$ with a guaranteed purity of $98 \%$, also containing $10 \mathrm{mg}$ of piperine/ capsule) or an inert placebo (methyl cellulose) once daily, with the treatment allocation dictated by Latin square. Participants were instructed to consume their daily capsule in the morning and preferably with breakfast.

Participants consumed their first and last capsule of treatment during the two laboratory visits and were instructed to selfsupplement every day in the interim. Participants kept a treatment log during this time, noting down the time of capsule consumption every day. A treatment pot containing thirty-two capsules was given to each participant at the end of visit 1, which was enough for $28 \mathrm{~d}$ of supplementation plus extra in case of loss, continued supplementation because of unforeseen circumstances and to verify compliance.

All treatments were administered in identical green vegetarian capsules with the Biotivia ${ }^{\mathrm{TM}}$ logo and presented in identical white treatment pots with only the participant number to identify them. All treatments were produced by Biotivia ${ }^{\mathrm{TM}}$, prepared by the lead investigator and coded by a third party who had no further involvement in any aspect of the study. No member of the investigational team was aware of the contents of the capsules until a blind-data review was completed.

Measures of cerebral blood flow (CBF)

Two complementary techniques were used as follows.

Acute changes in CBF-NIRS: NIRS is non-invasive brain imaging technique predicated on the absorption by oxygenated $\mathrm{HB}$ (oxy-Hb) and deoxygenated $\mathrm{Hb}$ (deoxy-Hb) of differing wavelengths of IR light, introduced through the intact scalp/ skull. CW-NIRS can be used to assess acute changes in local $\mathrm{CBF}$, as indexed by concentration changes in total $\mathrm{Hb}$ during a

Table 1. Participant composition*

\begin{tabular}{|c|c|c|c|c|c|}
\hline Measure (number of participants) & Female/male $(n)$ & Mean age (years) & Age range (years) & Right handed/left handed $(n)$ & Placebo/resveratrol $(n)$ \\
\hline Overall recruited ( $n 60$ ) & $51 / 9$ & 20.52 & $18-29$ & $53 / 7$ & $30 / 30$ \\
\hline Cognitive performance ( $n$ 41) & $36 / 5$ & 20.00 & $18-27$ & $35 / 6$ & $19 / 22$ \\
\hline NIRS $(n 46)$ & $39 / 7$ & $20 \cdot 45$ & $18-29$ & $39 / 7$ & $24 / 22$ \\
\hline $\operatorname{TCD}(n 46)$ & $40 / 6$ & 20.08 & $18-29$ & $40 / 6$ & $21 / 25$ \\
\hline Blood pressure (n 24) & $21 / 3$ & $20 \cdot 75$ & $18-29$ & $21 / 3$ & $15 / 9$ \\
\hline $\mathrm{GHQ}(n 53)$ & $45 / 8$ & $20 \cdot 17$ & $18-29$ & $46 / 7$ & $28 / 25$ \\
\hline POMS ( $n$ 54) & $46 / 8$ & 20.07 & $18-29$ & $47 / 7$ & $28 / 26$ \\
\hline PSQI (n 53) & $45 / 8$ & $20 \cdot 15$ & $18-29$ & $47 / 6$ & $28 / 25$ \\
\hline Food consumption ( $n$ 55) & $47 / 8$ & $20 \cdot 15$ & $18-29$ & $48 / 7$ & $29 / 26$ \\
\hline Treatment guess ( $n$ 57) & $49 / 8$ & $20 \cdot 25$ & $18-29$ & $50 / 7$ & $28 / 29$ \\
\hline
\end{tabular}

NIRS, near-IR spectroscopy; TCD, transcranial Doppler; GHQ, General Health Questionnaire; POMS, Profile of Mood States; PSQI, Pittsburgh Sleep Quality Index.

* In all, sixty participants were originally recruited to take part in all aspects of assessments apart from the blood pressure measurement, which utilised only thirty participants because of the potential disruption this may have caused to NIRS measurement. Reasons for excluding data from analyses include: technical problems with equipment (affecting aspects of twelve cognitive performance data sets, fourteen NIRS, fourteen TCD recordings (namely not being able to locate a consistent, 5-min, blood flow trace in the latter and data which were outside of the calculated standard deviations of this cohort, and may suggest an ill-fitting headband, with regards NIRS) and six blood pressure readings) and participants not complying with proper completion of measures/omitting to respond (affecting aspects of seven cognitive performance data sets, seven responses from the GHQ, six from the POMS, seven from the PSQI, five from the food consumption questionnaire and three from the treatment guess response). 
single continuous recording session; see Kennedy et al. ${ }^{(2)}$ for a full description of the methods used here. Given that CW-NIRS generates concentration change data that is intrinsically baseline-adjusted to the concentration immediately before the first data point in the recording session, it cannot be used to quantify gross changes in $\mathrm{CBF}$ parameters that take place between two separate recording sessions. In this instance, the change from baseline data generated by the NIRS system was subjected to a second baseline adjustment by creating 'change from baseline' data with respect to the $10 \mathrm{~min}$ of NIRS data collected immediately before the treatment; this provided a more accurate baseline measure of immediately pre-treatment NIRS parameters. All subsequent NIRS data were collapsed into 2-min epochs (twenty resting-period epochs spanning 0-40 min and twenty task-period epochs spanning 40-80 min).

Chronic changes in CBF-TCD: given the inability of $\mathrm{CW}-\mathrm{NIRS}$ to measure chronic changes in CBF parameters, a second measure of CBF was also used. TCD sonography is a non-invasive method of measuring CBFV through the basal intracerebral vessels through the intact skull ${ }^{(20)}$, and it was used at pre- and post-dose time points on day 1 and day 28. Pulses of ultrasound penetrate the skull at a number of 'acoustic windows', which include temporal, orbital, foraminal and submandibular, insonating vessels at particular depths, with the returning 'echo' displayed as a Doppler waveform ${ }^{(21)}$. The mean velocity, peak systolic velocity, diastolic velocity and pulsatility index (all centimetre per second) of the insonated vessel are provided, indicating the speed of the flow of blood and the variability of blood velocity.

TCD has been used to investigate blood flow abnormalities in a number of haematological, for example, stroke risk in sickle cell patients ${ }^{(22)}$, and vascular, for example, cerebrovascular reactivity in degenerative and vascular dementia ${ }^{(23)}$, disorders, as well as investigating the relationship between brain activity (in response to cognitive tasks) and blood flow velocity in healthy participants ${ }^{(24)}$ and the $\mathrm{CBFV}$ response to pharmacological interventions, for example, caffeine ${ }^{(25)}$, and drugs, for example, in cocaine abusers ${ }^{(26)}$.

In the current study, CBFV was measured with participants sitting in a reclined position in a quiet room. A trans-temporal acoustic window was used for assessment of the right MCA using pulsed TCD (Digi-Lite ${ }^{\text {TM }}$; Rimed) with a $2-\mathrm{MHz}$ probe held in place by a light, mounted head frame. This device provides mean velocity, peak systolic velocity, diastolic velocity and pulsatility index information every $30 \mathrm{sec}$, equating to approximately 10 values across the 5-min recording used here, for each of the four aforementioned variables. These were averaged to give 1 value for that time point (before and after dose on day 1 and before and after dose on day 28) for statistical analysis.

\section{Cognitive tasks}

The computerised battery of cognitive tasks (which all, to a greater or lesser extent, activate the prefrontal cortex: serial subtractions ${ }^{(27)}$; rapid visual information processing $(\mathrm{RVIP})^{(28)}$; 3 -Back ${ }^{(29)}$ ) were delivered on a laptop using the Computerised Mental Performance Assessment System (COMPASS, University of Northumbria) software, and they comprised serial subtractions (2 min each of serial 7, 13 and 17s) RVIP (2 min)

Both the serial subtraction and RVIP task are described in detail in Kennedy et al. ${ }^{(2)}$.

3-Back: the 3-Back version of this task was used in this paradigm, and it required participants to indicate whether the letter presented on screen was also present three letters back in the letter sequence. Participants must respond by pressing the 'yes' or 'no' button on the response box, to each letter, as quickly as they can. This task lasts for $2 \mathrm{~min}$, and it is scored for accuracy and reaction time.

\section{Questionnaires}

Food consumption questionnaire. A non-validated food consumption questionnaire was used to collect information on the general diet of participants (e.g. 'How many portions of fruit and vegetables did you eat on an average day in the past week?') and specifically polyphenol/resveratrol consumption (e.g. 'In the entire previous week, on how many occasions have you eaten a portion of berries or grapes?'). The questionnaire consisted of thirteen questions, with several questions also relating to compliance (e.g. "Was treatment consumed with breakfast and/or before 9:30 am every day in the past week?') and medication ('Have you consumed any medication in the past week? If so, please state the medication, dose, when taken and for what reason'). This researcher-created questionnaire has no reliability/sensitivity measures, and it was used solely as a tool to detect any gross changes in the consumption patterns of participants, which might affect outcome measures. The researcher noted no salient dietary or medication changes across the study for any of the participants.

General Health Questionnaire. The General Health Questionnaire $(\mathrm{GHQ})^{(30)}$ used in the current study was the twenty-eight-item scaled version, which assesses somatic symptoms, anxiety and insomnia, social dysfunction and severe depression. The twenty-eight items are scored from 0 to 3, with participants indicating the frequency or extent to which they have experienced a number of issues, such as 'Have you recently been having hot or cold spells?', in the previous week. The items combine to assess the four aforementioned sub-scales, and the total possible score (when these four sub-scales are collated) ranges from 0 to 84, with higher scores representing more negative symptoms.

Profile of Mood States. The Profile of Mood States (POMS) is a well-validated questionnaire of mood states and their fluctuations both in the clinical and research settings ${ }^{(31)}$. Participants rated sixty-five adjectives (e.g. unhappy, considerate), in terms of how much they had felt each one in the past week, using a five-point scale from 'not at all' to 'extremely'. Scores from these sixty-five items (which includes seven dummy adjectives) are combined to give six global scores of 'tension', 'depression', 'anger', 'fatigue', 'confusion' and 'vigour'. A total mood 
disturbance score can also be calculated by adding the scores from the first five of these global scores and subtracting 'vigour'.

Pittsburgh Sleep Quality Inventory. The Pittsburgh Sleep Quality Inventory (PSQI) is a well-validated subjective measure of the quality and pattern of sleep ${ }^{(32)}$. The current study tailored this questionnaire to assess sleep during the past 'week' rather than 'monthly' as per the original. The PSQI assesses seven factors - subjective sleep quality, sleep latency, sleep duration, habitual sleep efficiency, sleep disturbances, use of sleep medication and daytime dysfunction - via questions regarding sleep timings and zero to three-point scales in which participants rate whether they have experienced a number of issues (e.g. 'During the past week, how often have you had trouble sleeping because you have had bad dreams?') from 'not during the past week' to ' 3 or more times in the past week'. A global sleep score is created by totalling the seven subfactor scores, with higher scores indicating poorer sleep quality.

\section{Treatment guess}

During the day 28 visit, participants were asked to guess which treatment they thought they had been taking for the duration of the study and to explain any reasons for that guess.

\section{Procedure}

This investigation required participants to attend the laboratory for an initial training/screening session and then on two separate occasions, $28 \mathrm{~d}$ apart, for laboratory-based testing sessions. Participants were required to supplement themselves with one capsule per $\mathrm{d}$ in the interim.

Upon arrival at both day 1 and day 28, laboratory visit participants completed four questionnaires: a food consumption questionnaire; the GHQ; POMS; and the PSQI. All questionnaires were answered in relation to the previous $7 \mathrm{~d}$ and completed every $7 \mathrm{~d}$ during the supplementation period. After filling in the questionnaires, participants then gave a blood pressure (BP) reading or an intravenous blood sample (fifteen participants provided blood samples; see below for more information and the demographics of the seven participants from the resveratrol condition entered into the analysis), which was immediately followed by a 5-min rest. A 5-min recording of cerebral perfusion in the MCA was then taken with TCD. The NIRS headband was then positioned onto the forehead of the participant to monitor $\mathrm{CBF}$ in the prefrontal cortex throughout the session. Once a reliable trace was identified, participants commenced $20 \mathrm{~min}$ ( $\times 2$ repetitions of the battery) of baseline cognitive tasks. The first of these repetitions acted as a 'refresher', attenuating any practice effects, and the second was used to create change from baseline data for the analysis of cognitive outcome data. A 10-min rest period then followed, with NIRS data averaged across this period and used as an accurate, immediately pre-treatment baseline for the calculation of change from baseline data for the post-treatment periods. During this 10-min resting period, participants watched a nonarousing DVD. Participants then consumed the first day's treatment and continued to watch the DVD for a further 40-min absorption period. After this period, a BP reading was taken in those who did not provide a blood sample previously and 40 min of post-dose tasks commenced. After completion of the task, a further BP reading was taken from the relevant participants, followed by a short break before the second TCD recording was conducted. After the TCD recording, participants were either free to leave the laboratory or provided a final blood sample if they were part of the aforementioned sub-section of participants. The timelines and running order of the testing sessions are shown in Fig. 1.

\section{Bioavailability assessment}

Participants. Complete sample sets comprising all four time points were obtained from fifteen participants (eight from placebo and seven from resveratrol; ten females and five males; mean age 19.87 years; range $18-25$ years). All participants were

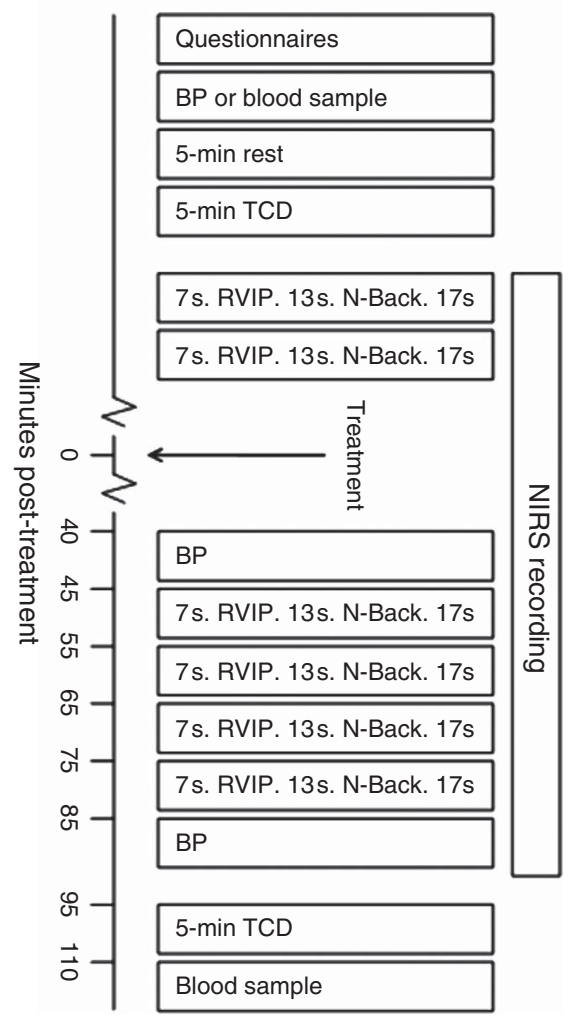

Fig. 1. Upon arrival, participants completed four questionnaires (a food consumption questionnaire, the General Health Questionnaire, Profile of Mood States and the Pittsburgh Sleep Quality Index), which they answered in relation to the previous $7 \mathrm{~d}$ and completed every $7 \mathrm{~d}$ during the supplementation period. Participants then gave a blood pressure $(\mathrm{BP})$ reading or an intravenous blood sample, which was immediately followed by a 5-min rest. A 5-min recording of cerebral perfusion in the middle cerebral artery was then taken with the transcranial Doppler (TCD). The near-IR spectroscopy (NIRS) headband was then positioned, and $20 \mathrm{~min}$ of baseline tasks commenced. A 10-min rest then followed, during which participants watched a non-arousing DVD. Participants then consumed their treatment capsule and continued to watch the DVD for a further 40-min absorption period. A BP reading was then taken from a subsample of participants and $36 \mathrm{~min}$ of post-dose tasks commenced. The NIRS headband was removed and a further BP reading taken, followed by a short break, before the second TCD recording was conducted. Following the TCD recording, the aforementioned sub-section of participants provided a blood sample and left the laboratory. RVIP, rapid visual information processing. 
asked, at the beginning of the study, if they would provide blood samples as part of the investigation: the above fifteen participants represent those who agreed to this aspect of the study and for whom all four samples could be collected in full. The seven resveratrol participants included in the analysis comprised six females and one male, with a mean age of 19.43 years, ranging from 18 to 21 years.

Venous blood samples were collected before consuming the day's treatment, and $110 \mathrm{~min}$ post dose in this sub-sample of participants using 4.7- $\mathrm{ml}$ monovettes (containing lithium heparin) (Sarstedt AG \& Co.). Samples were centrifuged at $2500 \mathrm{rpm}$ for $15 \mathrm{~min}$ at $20^{\circ} \mathrm{C}$ to yield plasma, which was then stored at $-80^{\circ} \mathrm{C}$ until analysis.

The preparation of samples and LC-MS analysis are as per a previous study conducted by this laboratory ${ }^{(33)}$.

\section{Statistics}

The analyses of TCD, plasma, questionnaire, behavioural and treatment guess data were conducted with IBM SPSS Statistics 19.0 for Windows (SPSS Inc.). NIRS data were analysed with Minitab 16 for Windows (Minitab Inc.).

Questionnaire data analysis. Questionnaire data (GHQ, POMS and PSQI) for each of the four post-dose weekly completions were analysed as change from baseline (the questionnaire scores obtained on day 1 before treatment) for each individual variable/sub-component by a mixed (day $(\times 4)$ : 7, 14, 21, 28, by treatment $(\times 2): 500 \mathrm{mg}$ resveratrol and placebo) ANOVA with Bonferroni-corrected post hoc Student's $t$ tests conducted if a significant main and/or interaction effect was evinced here.

Treatment guess analysis. Treatment guess data were analysed by $\chi^{2}$ test.

Transcranial Doppler. The raw data for each of the four TCD variables (mean velocity, peak systolic velocity, diastolic velocity and pulsatility index) were analysed by a mixed (treatment $(\times 2)$ : $500 \mathrm{mg}$ resveratrol and placebo, by time $(\times 4)$ : baseline day 1, post-dose day 1, pre-dose day 28 and post-dose day 28) ANOVA.

Plasma analysis. The raw data for each of the four forms of plasma resveratrol (resveratrol-3-sulphate, resveratrol-4'-O-glucuronide, resveratrol-3'-O-glucuronide and 'total metabolites'; which is the sum of the three metabolites) were analysed via ANOVA with time as a factor ( $\times 4$ : baseline day 1 , post-dose day 1, pre-dose day 28 and post-dose day 28).

Cognitive task data and blood pressure analysis. The cognitive task and BP measures produce data that can be analysed to assess both acute (potential treatment effects within day 1), pure-chronic (chronic treatment-related effects that have taken place across the 28-d supplementation period but before taking the day 28 treatment) and superimposed acute/chronic (the difference in 'acute' effects between day 1 and day 28) effects of resveratrol. To adequately analyse the 'acute', 'pure chronic' and 'superimposed acute/chronic' effects of the treatments, two separate ANOVA were conducted:

1. Pure chronic effects

To ascertain whether any pure chronic effects of resveratrol supplementation had taken place, pre-dose data on day 28 were converted to change from day 1 pre-dose baseline and analysed via one-way ANOVA to compare performance between treatments.

2. Acute, chronic and superimposed effects

To ascertain whether any acute and/or superimposed chronic effects of resveratrol supplementation had taken place, data were converted to change from baseline with respect to the pre-treatment scores on the first day of treatment (day 1) and analysed via a repeated measures ANOVA (treatment (resveratrol/placebo, $\times$ repetition $(\times 4$ for cognitive data and $\times 2$ for BP), by day (day $1 / 28$ )).

Both ANOVA were used in order to tease apart acute effects restricted to day 1 (treatment $\times$ day interactions with significant effects restricted to day 1 ), acute effects across both day 1 and day 28 (main effect of treatment and/or a treatment $\times$ repetition interaction) and a superimposed acute/chronic effect (treatment $\times$ day interaction with significant effects restricted to day 28 (interpreted with reference to the pure chronic ANOVA results)). If any such main and/or interaction effects were observed, then Bonferroni-corrected post hoc Student's $t$ tests were conducted to assess where these differences lie. This analysis plan has proven sensitivity in detecting the acute and chronic effects of ginseng in healthy, human participants previously ${ }^{(34)}$.

Near-IR spectroscopy analysis. NIRS data were converted to 'change from baseline' (calculated from the 10-min pre-treatment resting period) and averaged across 2-min epochs during the 40-min 'rest/absorption' and 40-min cognitive task performance period. ANOVA (treatment group $\times 2$-min epoch $\times$ day) was conducted on these data, with planned comparisons of data from each epoch being made between placebo and $500 \mathrm{mg}$ of resveratrol (resulting in forty planned comparisons for oxy-HB, deoxy-Hb and total-Hb) using $t$ tests calculated with the mean squares error from the ANOVA ${ }^{(35)}$. A significant result on this ANOVA was not used as a prerequisite for carrying out and interpreting the planned comparisons, and it is therefore not presented here. However, in order to reduce the potential for type I errors, all planned comparisons were Bonferroni corrected, and only those planned comparisons associated with a consistent pattern of significant effects are interpreted and reported herein.

\section{Results \\ Compliance}

Potential compliance ranged from 0 to $114 \%$ (the upper limit reflecting thirty-two capsules consumed over $28 \mathrm{~d}$ ). Average compliance was $101 \%$, with a range of $78.5-114.3 \%$. Data from one participant with $78.5 \%$ compliance (who provided blood samples in the placebo condition only) were excluded from 
analysis, because they were below a pre-set level of $80 \%$, making average compliance $101.4 \%$ with a range of $92 \cdot 9-114.3 \%$.

\section{Treatment guess}

$\chi^{2}$ test revealed no significant difference between treatment guesses in the two treatment groups: $\chi^{2}=0 \cdot 766 ; \mathrm{df}=1 ; P=381$.

\section{Near-IR spectroscopy parameters}

Total $\mathrm{Hb}$. Planned comparisons revealed that, on day 1 , levels of total-Hb were significantly higher after resveratrol, compared with placebo, during the 2-min epochs spanning 35-38 min post dose $(35 / 36 \mathrm{~min}(P=0.003), 37 / 38 \mathrm{~min}(P=0.008))$ in the absorption period and the epochs spanning 75-78 min $(75 /$ $76 \mathrm{~min}(P=0.008), 77 / 78 \mathrm{~min}(P=0.005))$ of the post-dose task period. No significant differences were found between resveratrol and placebo on day 28.

Oxygenated $\mathrm{Hb}$. Planned comparisons revealed that, on day 1 , levels of oxy-Hb were significantly higher in the resveratrol condition, compared with placebo, during the 2-min epochs commencing 23 ( $P=0.002), 27(P=0.005), 33$ ( $P=0.002), 35$ $(P=0.001)$ and $37(P=0.009)$ min post dose in the absorption period and the epochs spanning 41-44 (41/42 $(P=0.006)$, 43/44 ( $P=0.001)$ ), 53-54 ( $P=0.001), 61-68(P=0.0008,0.001$, 0.007 and 0.001 , respectively $), 71-72(P=0.003), 75-78(75 / 76$ $(P=0.0002)$ and $77 / 78(P=0.0002))$ min of the post-dose task period. No significant differences were found between resveratrol and placebo on day 28.

Deoxygenated $\mathrm{Hb}$. Planned comparisons revealed that, on day 1 , levels of deoxy-Hb were significantly higher in the placebo condition, compared with resveratrol, during the 2-min epochs commencing 27 ( $P=0.001), 29(P=0.006)$ and $35(P=0.003) \mathrm{min}$ post-treatment in the absorption period and the epochs commencing $43 \mathrm{~min}(P=0.004)$, and spanning 51-54 $\mathrm{min}$ (51/52 $\mathrm{min}$ $(P=0.0002), 53 / 54 \mathrm{~min}(P=0.004))$, and those spanning $61-72$ $\min (61 / 62 \min (P=0.001), 63 / 64 \mathrm{~min}(P=0.003), 65 / 66 \mathrm{~min}$ $(P=0.0005), 67 / 68 \mathrm{~min}(P=0.002), 69 / 70 \mathrm{~min}(P=0.004), 71 / 72$ $\min (P=0.0008)$, respectively) and those spanning 75-80 $\mathrm{min}(75 /$ $76 \min (P=0.001), 77 / 78 \min (P=0.003), 79 / 80 \min (P=0.004)$, respectively) min of the post-dose task period. No significant differences were found between resveratrol and placebo on day 28.

Mean total-, oxy- and deoxy-Hb levels for placebo and resveratrol, across day 1 and day 28, are shown in Fig. 2.

\section{Transcranial Doppler parameters}

No significant acute chronic or gross chronic effects were observed with any of the four TCD parameters (Mean velocity, peak systolic velocity, diastolic velocity and pulsatility index).

\section{Cognitive task performance}

1. Pure chronic ANOVAThe results of the ANOVA on day 28 pre-dose data (converted to change from day 1 baseline) comparing performance between $500 \mathrm{mg}$ of resveratrol and placebo demonstrated a significant effect of treatment for the 3-Back task in terms of the percentage of correct responses $\left(F_{1,40}=8.60 ; P=0.006\right)$, with better performance in the resveratrol condition as compared with placebo.

2. Acute, chronic and superimposed ANOVA

The results of the treatment $\times$ repetition $\times$ day ANOVA are as follows. Note that, for brevity, only those significant main and/or interaction effects involving treatment are described here, but see online Supplementary Materials for all ANOVA $F$ and $P$ value tables.

$7 \mathrm{~s}$ incorrect. Analysis revealed a main effect of treatment $\left(F_{1,39}=6.40 ; P=0.016\right.$ ) (with the mean for number of serial $7 \mathrm{~s}$ incorrect responses for placebo, overall, higher than the mean for $500 \mathrm{mg}$ resveratrol) and a day $\times$ repetition $\times$ treatment interaction $\left(F_{3,117}=0.260 ; P=0.034\right)$. Post boc comparisons (Bonferroni corrected) revealed a significant difference on day 1 at repetition $4(P=0.005)$ and trends for differences on day 1 at repetition $2(P=0.073)$ and on day 28 at repetition 3 $(P=0 \cdot 070)$. The mean number of incorrect responses was lower in the 500-mg resveratrol condition in all three cases.

17s correct. The ANOVA revealed an interaction between day $\times$ treatment $\times$ repetition $\left(F_{3,117}=3.45 ; P=0.019\right)$. Post boc comparisons revealed significant differences on day 28 at repetition 1 and repetition 3 (both $P=0.04$ ), with the mean number of serial $17 \mathrm{~s}$ correct completions being higher in the placebo condition in both cases.

17s incorrect. The ANOVA showed a main effect of treatment $\left(F_{1,39}=5.79 ; P=0.021\right.$ ) (with the mean number of $17 \mathrm{~s}$ subtraction incorrect responses, overall, being higher in the placebo condition as compared with $500 \mathrm{mg}$ of resveratrol). An interaction between repetition $\times$ treatment $\left(F_{3,117}=3.55\right.$; $P=0.017)$ was also observed. With regard to the repetition $\times$ treatment interaction, post boc comparisons revealed only one significant comparison between treatments at the fourth repetition on day 28. Here, the mean number of incorrect responses was higher $(P=0.003)$ in the placebo condition.

\section{General health}

There were no significant treatment-related differences on the GHQ or its subcomponents.

\section{Sleep}

There were no significant treatment-related differences on the PSQI or its subcomponents.

\section{Mood}

A significant treatment effect was observed for the 'fatigue' measure alone $\left(F_{1,52}=9 \cdot 37 ; P=0.003\right)$, which was derived from the POMS questionnaire. Further analysis with Bonferronicorrected post hoc Student's $t$ tests demonstrated that subjective ratings of fatigue were significantly lower for resveratrol on day $7(P=0.04)$, day $21(P=0 \cdot 013)$ and day $28(P=0.001)$. A move towards a trend was also evinced for day $14(P=0.097)$. 
Day 1
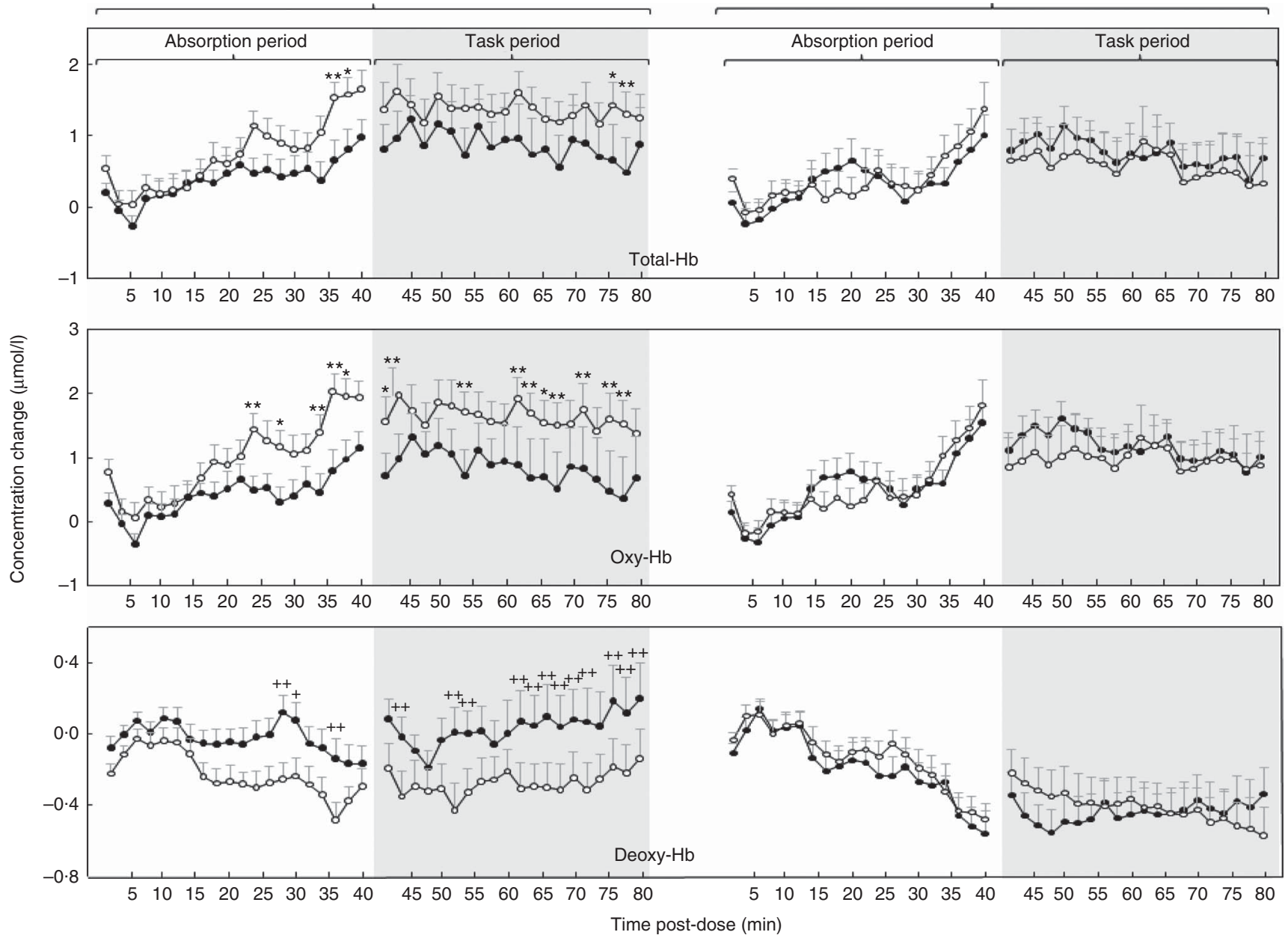

Fig. 2. Concentration changes from baseline in levels of (top) total $\mathrm{Hb}$ (Total-Hb), (middle) oxygenated $\mathrm{Hb}(\mathrm{Oxy}-\mathrm{Hb}$ ) and (bottom) deoxygenated $\mathrm{Hb}(\mathrm{Deoxy}-\mathrm{Hb})$ averaged across two-min epochs during a 40 -min absorption period and subsequent $40 \mathrm{~min}$ of cognitive task performance following placebo or $500 \mathrm{mg}$ of resveratrol on day 1 and day $28(n 46)$. —- Placebo; $-0 \_, 500 \mathrm{mg}$ of resveratrol. Values are means, with standard errors represented by vertical bars. Significance planned comparisons (Bonferroni corrected) between resveratrol and placebo of data from each 2-min epoch: * $P<0.05$ and ${ }^{* *} P<0.01$.

See online Supplementary Materials for average weekly ratings on POMS questionnaire and ANOVA $F$ and $P$ value tables.

\section{Blood pressure}

\section{Pure chronic ANOVA}

The results of the ANOVA on day 28 pre-dose BP measurements (converted to change from day 1 baseline) comparing readings between $500 \mathrm{mg}$ of resveratrol and placebo demonstrated only a significant effect for diastolic BP $\left(F_{1,28}=5 \cdot 86 ; P=0 \cdot 022\right)$, with levels being higher in the resveratrol condition.

2. Acute, sub-chronic and superimposed ANOVA

No significant effects were observed for systolic BP or heart rate. For diastolic BP, a significant interaction between treatment $x$ day was evinced $\left(F_{1,22}=6.61 ; P=0.017\right)$, which revealed only one significant comparison in the placebo condition between day 1 and day 28 at the 40-min post-dose measurement $(P=0 \cdot 46)$. Here the mean was higher overall on day 28 compared with day 1 .
See online Supplementary Materials for BP values and ANOVA $F$ and $P$ value tables.

\section{Plasma analysis (total metabolite levels)}

$6 \mathrm{~A}$ significant effect of time was observed $\left(F_{1 \cdot 35,8 \cdot 10}=7 \cdot 50\right.$; $P=0.02$ ) for levels of total resveratrol metabolites (the sum of resveratrol 3-O-sulphate and resveratrol-4'- and 3'-O-glucuronides), with pair wise comparisons revealing that day 1 postdose levels were higher than day 1 baseline $(P=0.023)$, that day 28 pre-dose levels were higher than day 1 baseline $(P=0.033)$ and that day 28 post-dose levels were higher than both day 1 baseline $(P=0.003)$ and day 28 pre-dose levels $(P=0.005)$. All three metabolites followed this same pattern of significance, and thus, for brevity, only total metabolite levels are reported here.

No resveratrol (in any form) was found in baseline samples on day 1 , indicating that all volunteers did not consume resveratrol-containing products before the study. No aglycone resveratrol was quantifiable in plasma at any time point, on either day. Resveratrol 3-O-sulphate was the predominant metabolite in all volunteers, contributing $73-77 \%$ of total 


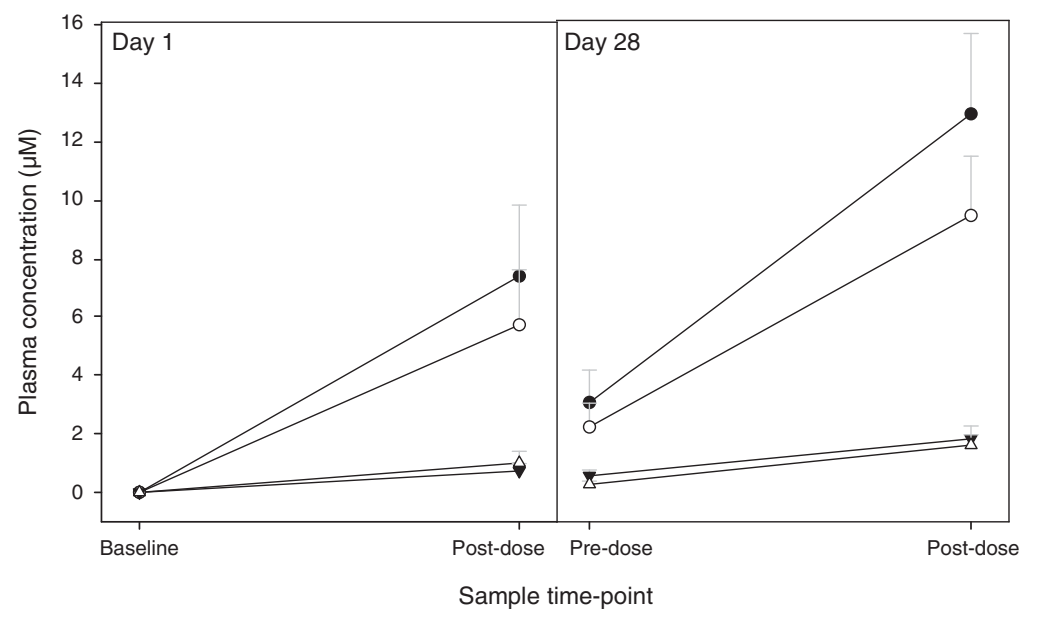

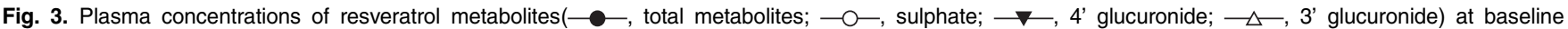
and post-dose (110 min post-administration of $500 \mathrm{mg}$ trans-resveratrol) on day 1 and day 28 . Values are means $(n)$ ), with standard errors represented by vertical bars.

metabolites. The 4'- and 3'-O-glucuronide forms evinced roughly equal contributions to the remaining metabolites in circulation.

Mean plasma concentration values ( $\mu \mathrm{m})$ for resveratrol metabolites at baseline and post-dose (110 min after administration) on day 1 and, after daily 500-mg consumption, on day 28 are shown in Fig. 3.

\section{Discussion}

In summary, the results here show that although a single dose of $500 \mathrm{mg}$ of trans-resveratrol can modulate CBF parameters in the frontal cortex in a pattern consistent with increased blood flow, supplementation for $28 \mathrm{~d}$ does not result in any clear improvements in cognitive function, despite an increase in plasma metabolite levels. However, there was evidence of significantly reduced fatigue and higher diastolic BP following extended supplementation. No modulation of subjective sleep quality, health or chronic CBF was observed.

The chronic 28-d dosing paradigm used in the current paper was designed to address the potential ineffectiveness of resveratrol at eliciting cognitive performance effects after acute, bolus supplementation ${ }^{(2,33)}$. The hypothesis was that chronic consumption of resveratrol might increase exposure to resveratrol - a polyphenol with known low bioavailability following acute administration ${ }^{(9)}$. This increased exposure may be expected to enhance the biological activity of resveratrol, specifically, of importance here, those with direct and/or indirect effects on cognitive function. However, analysis demonstrated that the only cognitive task measure to evince a pure chronic effect (derived by the comparison of changes in performance between resveratrol and placebo between day 1 baseline and day 28 before dose) was N-Back \% correct: that is, after $28 \mathrm{~d}$ of supplementation, participants in the 500-mg resveratrol condition completed significantly more correct 3-Back responses before taking their day's treatment, as compared with placebo. No effects on this measure were observed after consumption of treatment on day 1 or day 28 , nor were any effects observed on the other accuracy submeasure assessed here. The results of acute and chronic/ superimposed analysis revealed that, on day 28 , participants in the resveratrol condition performed slower, achieving less correct responses on the serial 17 subtractions task. However, on day 1 and day 28, participants in the same condition also performed more accurately (less incorrect responses) on the serial 7 and serial 17 subtraction tasks. Although these results suggest a speed accuracy trade-off, closer inspection of these significant main effects highlights an inconsistent and difficultto-interpret pattern, with the effects on the serial 7 task restricted to the fourth task battery repetition on day 1 only and the first, third and fourth repetitions, on day 28 , for the effects on the serial 17 subtraction task, where both higher and lower performance was seen in the resveratrol condition. Because of the lack of any clear pattern of results in both the acute and chronic effects of resveratrol on cognition here (and indeed the previous two studies assessing the effects of resveratrol on cognitive function), it is important to regard these results with caution. It may be that the relatively small sample here is masking a real effect, or a clearer effect, of resveratrol or it may be that a number of type I errors have inflated expectations. Nevertheless, only a tightly controlled, cross-over study with greater power would be able to address this issue.

The current study demonstrates that $500 \mathrm{mg}$ of trans-resveratrol is able to augment the CBF response to cognitive task demands, relative to placebo, after acute, oral administration to healthy human participants. This acute augmentation manifested in small, significantly higher levels of total-Hb, indicative of increased CBF, at the ends of the absorption- and post-dose task periods and a consistent pattern of significantly higher levels of oxy-Hb across some of the absorption- and post-dose task periods following the first dose of resveratrol on day 1. Levels of deoxy-Hb were also significantly lower in the resveratrol condition, as compared with placebo. This latter 
finding is directly opposite to that reported previously ${ }^{(2,33)}$, and it is contrary to the hypothesis that resveratrol would facilitate increased oxygen extraction because of its reported effects on oxidative phosphorylation $^{(36)}$. No clear reason for this anomalous finding can be offered at present, but it may be notable that although the previous two aforementioned resveratrol/ NIRS studies by this laboratory were cross-over studies the current study is the first to use a between-subjects design, and this may introduce an unanticipated degree of variability in $\mathrm{CBF}$ parameters. In contrast to day 1 , the consumption of the resveratrol treatment on day 28 was not found to have an acute effect on any of the CBF parameters. As noted above, CW-NIRS generates concentration change, rather than quantitative data, and therefore it only provides a measure of acute changes in haemodynamics during each discrete recording session. It therefore provides no direct measure of any changes that have taken place between recording sessions, in this case as a consequence of chronic resveratrol supplementation. The lack of an effect here may then reflect several distinct possibilities. It may, of course, reflect a simple attenuation of the acute effects seen following the first dose of resveratrol on day 1 . However, it could equally reflect either the raised levels of resveratrol metabolites seen before treatment on day 28 , which may have precluded a further acute effect of an additional dose on day 28 , or it may indicate that a gross (undetected) change in CBF parameters had already taken place, attenuating the possibility of any additional acute effects of the day 28 treatment.

In the current study, TCD was also incorporated to provide a measure of chronic CBF. This technique provides an absolute quantitative measure of $\mathrm{CBF}$ (in this case as indexed by $\mathrm{CBFV}$ in the right $\mathrm{MCA}$ ), which was intended to elucidate any gross chronic changes in $\mathrm{CBF}$ as a consequence of resveratrol supplementation. No significant changes in CBFV were observed with TCD, suggesting a simple absence of modulation of $\mathrm{CBF}$ by resveratrol. However, this interpretation should be tempered by several considerations. The first is that the recording period was much shorter (at $5 \mathrm{~min}$ ) than for NIRS, and it was undertaken entirely at rest, with no data collected during the period of task performance, during which resveratrol has been shown to have its most pronounced effects. Second, although the NIRS was used to measure local changes in CBF in the upper layers of the frontal cortex during tasks that activate this brain area, the right MCA supplies the entire right side of the cortex. Given this, any vasodilatory effects restricted to the locality of neural activity (in this case the prefrontal cortex) may have been swamped in the gross blood flow. Potential reasons for a lack of significant $\mathrm{CBFV}$ changes include the relatively short recording period with the TCD: 5 min, yielding only two measurements per minute, which may simply be to narrow a window to detect effects. The TCD recording periods were also conducted during times of minimal cognitive demand (pre and post the cognitive task periods) and, as such, metabolic substrate demands would have been less during these periods and an increase in the haemodynamic response unnecessary. Ideally, the TCD and NIRS would both have been used to record concomitantly throughout the absorption and cognitive task periods. Unfortunately, because of the physical constraints of the equipment used here, this was not possible.
The current study does, however, report vascular effects of resveratrol in the periphery on day 28 , with the analysis of pure chronic effects (derived by comparing change from day 1 baseline BP measurements between resveratrol and placebo to pre-treatment on day 28) demonstrating higher diastolic BP in resveratrol-supplemented participants. No pre-treatment baseline differences in BP readings nor acute effects of treatment within day 1 or day 28 were observed. This finding is intuitively unexpected, as resveratrol has previously been shown to be a vasodilator ${ }^{(6,37)}$ - a phenomenon associated with lowered BP. Whether resveratrol can act as a vasoconstrictor is, at present, unknown, but it may be noteworthy that structurally similar polyphenols, such as the tea polyphenol epigallocatechin-3-gallate (EGCG), can act both as both vasodilators and vasoconstrictors depending on the dose and the time of assessment ${ }^{(38)}$. EGCG has also been investigated with regard to its cognitive and CBF effects in humans, with a single dose of $135 \mathrm{mg}$ leading to a significant reduction in CBF as compared with placebo, which might indeed be suggestive of vasoconstriction.

No significant differences between treatments, or withintreatment changes, were observed with subjective perceptions of general health (as assessed by the GHQ) or sleep (as assessed by the PSQI). With regard to subjective perceptions of mood, the only variable on the POMS questionnaire that evinced any significant difference was 'fatigue', which remained significantly lower across the entire 28 -d period in the resveratrol condition, as compared with placebo. Little research exists regarding the effects of polyphenols on mood, but this anti-fatigue effect may find an explanation in in vitro and animal work, which reports the ability of resveratrol to inhibit MAO-A/B activity. This inhibition was reported to lead to an increase in monoamine neurotransmitter concentrations, namely 5-hydroxytryptophan (5-HT), noradrenaline and dopamine, with a concomitant improvement in mood, which was similar to that seen with imipramine and fluoxetine in mice ${ }^{(18)}$. Interestingly, quercetin, another red wine polyphenol, also shows anti-fatigue activity through increased energy expenditure and endurance capacity in mice ${ }^{(39,40)}$ and power output in elite male cyclists when part of a cocktail of supplemented compounds ${ }^{(41)}$. Mechanisms include increased blood flow because of vasorelaxation ${ }^{(42)}$ and oxygenation, with Davis et $a l^{(40)}$ also reporting SIRT-mediated increases in mitochondrial gene expression in brain and skeletal muscles. Both mechanisms are shared with resveratrol ${ }^{(36,42)}$ and could explain the increased energy levels seen here. It is worth noting here that, although there was no statistically significant difference in baseline (before dose on day 1) levels of fatigue between resveratrol and placebo participants, the baseline values were nevertheless numerically higher in the former group (8.04 compared with 5.54 , respectively), which might suggest that this effect represents a return to normal levels for the resveratrol group following an unusually high baseline.

Analysis of the plasma samples, taken from a sub-sample of seven participants from the resveratrol condition on day 1 , demonstrated increases in acute resveratrol metabolite levels post dose very similar to those seen in a previous study conducted by this laboratory ${ }^{(2)}$. Pre-dose levels of metabolites on 
day 28 were also significantly higher than pre-dose levels on day 1 , suggesting that chronic consumption results in an accumulation of resveratrol metabolites in plasma. They subsequently increased following the day 28 treatment, and again ended at a significantly higher level than that post dose on day 1. Pre- and post-dose levels of resveratrol on day 28 were significantly higher than baseline levels on day 1 and, within day 28, post-dose levels were significantly higher than pre-dose levels. Taken together, these findings suggest (hence their presence before treatment administration on day 28) that this may amplify the increase following acute administration (hence numerically higher levels at day 28 post dose compared with day 1 post dose). The fact that the day 1 baseline mean levels were 0 does render this comparison statistically problematic. However, disregarding statistical significance, the fact that metabolites were present on day 28 (considering that levels were 0 at baseline on day 1) is indicative that an increase in plasma levels of resveratrol had taken place. This novel finding of accumulating levels of resveratrol metabolites as a consequence of chronic administration certainly warrants further investigation with larger samples, as previous acute dose research does not suggest that plasma metabolites should still be present beyond $24 \mathrm{~h}^{(9)}$, or certainly not at the pre-dose levels seen here on day $28^{(10)}$. It may be possible that these effects are the result of some other, unknown, factor/s - for instance, the consumption by participants of more resveratrol-containing products or an additional resveratrol capsule before attending the laboratory on day 28. However, this seems unlikely, and is argued against by the participants' treatment diaries and a capsule count.

The methodology of the current study had a number of strengths and limitations. The nature of the paradigm, namely the time frame involved and the use of equipment that dictates individual testing (i.e. the NIRS and TCD), necessarily means that the sample size is somewhat restricted for outcome measures such as cognitive performance, which ideally require a larger sample than the physiological measures. In this study, the issue was exacerbated by the loss of a number of sets of data (due largely to an equipment failure), which reduced the number of cognitive performance data sets. This renders interpretation of the cognitive data more difficult, but an argued strength of this paper is the caution with which the authors have regarded such data. Another limitation relates to the equipment utilised here to measure CBF. As noted above, CW-NIRS only generates acute concentration change data, and therefore the question that it was used to address on day 28 of the current study was 'Are the acute haemodynamic effects of the single dose of resveratrol taken on day 28 the same, or different, to those seen following the first dose taken on day 1?'. The results showed that there were no acute effects on day 28 , and thus they were different. However, the difficulty in interpreting this finding further is that this could reflect an attenuation of the acute effects over time, but it could equally be the result either of the raised levels of resveratrol metabolites already seen before taking the day 28 treatment, or indeed unmeasured chronic effects on CBF. To address the last of these points, TCD was incorporated as a measure of chronic changes of absolute CBFV, but this measure showed no effect, although again this could be due to methodological issues (including measuring at rest, rather than during task performance, and the diffuse rather than local nature of the measurement). It would therefore be advantageous to revisit the question of the chronic effects of resveratrol on $\mathrm{CBF}$ using the more recently introduced 'quantitative' NIRS, which, as the name suggests, generates quantitative, rather than concentration change, data. In terms of strengths, the current paper incorporated a range of methodologies in order to answer the hitherto unaddressed question as to whether resveratrol can engender chronic cognitive effects. This is also the first paper to show that repeated consumption of resveratrol can lead to cumulative plasma levels at a dose that is recommended by many over-the-counter resveratrol products.

In conclusion, the current study reports that chronic, 28-d supplementation of $500 \mathrm{mg}$ of trans-resveratrol results in significantly reduced fatigue and higher diastolic BP, but it does not modulate sleep, health or chronic CBF. The single, chronic, cognitive effect evinced by resveratrol and the confusing pattern of acute effects should be treated with caution. This study is the first to suggest that chronic resveratrol consumption could result in cumulative plasma levels in healthy humans after oral administration.

\section{Supplementary material}

For supplementary material/s referred to in this article, please visit http://dx.doi.org/doi:10.1017/S0007114515003037

\section{Acknowledgements}

The direct study costs (participant payments and treatments) of the research described herein were funded by Biotivia ${ }^{\mathrm{TM}}$ Longevity Bioceuticals. Biotivia had no role in the design, analysis or writing of this article.

All of the authors (E. L. W., C. F. H.-R., J. L. R., G. W., T. D., W. Z. and D. O. K.) were actively involved in the planning of the research described herein and in writing the paper. E. L. W. collected the data. G. W., T. D. and W. Z. planned and carried out the analysis of the plasma samples. All authors contributed to and reviewed the final publication.

There are no conflicts of interest.

\section{References}

1. Fremont L (2000) Biological effects of resveratrol. Life Sci 66 , 663-673.

2. Kennedy DO, Wightman EL, Reay JL, et al. (2010) Effects of resveratrol on cerebral blood flow variables and cognitive performance in humans: a double-blind, placebo-controlled, crossover investigation. Am J Clin Nutr 91, 1590-1597.

3. Gresele P, Pignatelli P, Guglielmini G, et al. (2008) Resveratrol, at concentrations attainable with moderate wine consumption, stimulates human platelet nitric oxide production. J Nutr 138, 1602-1608.

4. Rivera L, Morón R, Zarzuelo A, et al. (2009) Long-term resveratrol administration reduces metabolic disturbances and lowers blood pressure in obese Zucker rats. Biochem Pharmacol 77, 1053-1063.

5. Rush JWE, Quadrilatero J, Levy AS, et al. (2007) Chronic resveratrol enhances endothelium-dependent relaxation but does not alter eNOS levels in aorta of spontaneously hypertensive rats. Ex Biol Med (Maywood) 232, 814-822. 
6. Wong R, Howe P, Buckley J, et al. (2011) Acute resveratrol supplementation improves flow-mediated dilatation in overweight/obese individuals with mildly elevated blood pressure. Nutr Metab Cardiovasc Dis 21, 851-856.

7. Moss MC, Scholey AB \& Wesnes K (1998) Oxygen administration selectively enhances cognitive performance in healthy young adults: a placebo-controlled double-blind crossover study. Psychopharmacology (Berl) 138, 27-33.

8. Scholey A, Harper S \& Kennedy D (2001) Cognitive demand and blood glucose. Physiol Behav 73, 585-592.

9. Walle T, Hsieh F, DeLegge MH, et al. (2004) High absorption but very low bioavailability of oral resveratrol in humans. Drug Metab Dispos 32, 1377-1382.

10. Boocock DJ, Faust GES, Patel KR, et al. (2007) Phase I dose escalation pharmacokinetic study in healthy volunteers of resveratrol, a potential cancer chemopreventive agent. Cancer Epidemiol Biomarkers Prev 16, 1246-1252.

11. Kuhnle G, Spencer JP, Chowrimootoo G, et al. (2000) Resveratrol is absorbed in the small intestine as resveratrol glucuronide. Biochem Biophys Res Commun 272, 212-217.

12. Wang L, Heredia A, Song H, et al. (2004) Resveratrol glucuronides as the metabolites of resveratrol in humans: characterization, synthesis, and anti-HIV activity. J Pharma Sci 93, 2448-2457.

13. Marier JF, Vachon P, Gritsas A, et al. (2002) Metabolism and disposition of resveratrol in rats: extent of absorption, glucuronidation, and enterohepatic recirculation evidenced by a linked-rat model. J Pharmacol Exp Ther 302, 369-373.

14. Gescher AJ \& Steward WP (2003) Relationship between mechanisms, bioavailibility, and preclinical chemopreventive efficacy of resveratrol: a conundrum. Cancer Epidemiol Biomarkers Prev 12, 953-957.

15. Ide K, Horn A \& Secher NH (1999) Cerebral metabolic response to submaximal exercise. J Appl Physiol 87, 1604-1608.

16. Jia Z, Zhu H, Misra BR, et al. (2008) EPR studies on the superoxide-scavenging capacity of the nutraceutical resveratrol. Mol Cell Biochem 313, 187-194.

17. Donnelly LE, Newton R, Kennedy GE, et al. (2004) Antiinflammatory effects of resveratrol in lung epithelial cells: molecular mechanisms. Am J Physiol Lung Cell Mol Physiol 287, 774-783.

18. Xu Y, Wang Z, You W, et al. (2010) Antidepressant-like effect of trans-resveratrol: involvement of serotonin and noradrenaline system. Eur Neuropsychopharmacology 20, 405-413.

19. Liu C, Li S, Liu T, et al. (2007) Transcriptional coactivator PGC-1alpha integrates the mammalian clock and energy metabolism. Nature 447, 477-481.

20. Markus HS (2000) Transcranial Doppler ultrasound. Br Med Bull 56, 378-388.

21. Nicoletto HA \& Burkman MH (2009) Transcranial Doppler series part II: performing a transcranial Doppler. Am J Electroneurodiagnostic Technol 49, 14-27.

22. Adams R, McKie V, Carl E, et al. (1997) Long-term stroke risk in children with sickle cell disease screened with transcranial Doppler. Ann Neurol 42, 699-704.

23. Vicenzini E, Ricciardi MC, Altieri M, et al. (2007) Cerebrovascular reactivity in degenerative and vascular dementia: a transcranial Doppler study. Eur Neurol 58, 84-89.

24. Harders A, Laborde G, Droste D, et al. (1989) Brain activity and blood flow velocity changes: a transcranial Doppler study. Int J Neurosci 47, 91-102.
25. Jones HE, Herning RI, Cadet JL, et al. (2000) Caffeine withdrawal increases cerebral blood flow velocity and alters quantitative electroencephalography (EEG) activity. Psychopharmacology (Berl) 147, 371-377.

26. Herning RI, King DE, Better WE, et al. (1999) Neurovascular deficits in cocaine abusers. Neuropsychopharmacology 21, 110-118.

27. Kazui H, Kitagaki H \& Mori E (2000) Cortical activation during retrieval of arithmetical facts and actual calculation: a functional magnetic resonance imaging study. Psychiatry Clin Neurosci 54, 479-485.

28. Coull J, Frith C, Frackowiak RSJ, et al. (1996) A fronto-parietal network for rapid visual information processing: a PET study of sustained attention and working memory. Neuropsychologia 34, 1085-1095.

29. Jansma JM, Ramsey NF, Coppola R, et al. (2000) Specific versus nonspecific brain activity in a parametric N-back task. Neuroimage 12, 688-697.

30. Goldberg D (1978) Manual of the General Health Questionnaire. Windsor: NFER Publishing.

31. McNair DM, Lorr M \& Droppleman LF (1971) Profile of Mood States. San Diego, CA: Educational and Industrial Testing Service.

32. Buysse DJ, Reynolds CF, Monk TH, et al. (1989) The Pittsburgh Sleep Quality Index: a new instrument for psychiatric practice and research. Psychiatry Res 28, 193-213.

33. Wightman E, Reay J, Haskell C, et al. (2014) Effects of resveratrol alone or in combination with piperine on cerebral blood flow parameters and cognitive performance in humans: a randomised, double-blind, placebo-controlled, crossover investigation. Br J Nutr 112, 203-213.

34. Reay JL, Scholey AB \& Kennedy DO (2010) Panax ginseng (G115) improves aspects of working memory performance and subjective ratings of calmness in healthy young adults. Hum Psychopharmacol 25, 462-471.

35. Keppel G (1991) Design and Analysis. Englewood Cliffs, NJ: Prentice Hall.

36. Lagouge M, Argmann C, Gerhart-Hines Z, et al. (2006) Resveratrol improves mitochondrial function and protects against metabolic disease by activating SIRT1 and PGC-1 alpha. Cell 127, 1109-1122.

37. Wong R, Berry N, Coates A, et al. (2012) Sustained improvement of vasodilator function by resveratrol in obese adults. J Hypertens 30, e70.

38. Alvarez E, Campos M, Justiniano H, et al. (2006) Study of the mechanisms involved in the vasorelaxation induced by (-)-epigallocatechin-3-gallate in rat aorta. Br Pharmacol 147, 269-280.

39. Stewart LK, Soileau JL, Ribnicky D, et al. (2008) Quercetin transiently increases energy expenditure but persistently decreases circulating markers of inflammation in C57BL/6J mice fed a high-fat diet. Metabolism 57, S39-S46.

40. Davis JM, Murphy EA, Carmichael MD, et al. (2009) Quercetin increases brain and muscle mitochondrial biogenesis and exercise tolerance. Am J Physiol Regul Integr Comp Physiolo 296, R1071-R1077.

41. MacRae HS \& Mefferd KM (2006) Dietary antioxidant supplementation combined with quercetin improves cycling time trial performance. Int J Sport Nutr Exerc Metab 16, 405--419.

42. Chen CK \& PaceAsciak CR (1996) Vasorelaxing activity of resveratrol and quercetin in isolated rat aorta. Gen Pharmacol 27, 363-366. 